ARTIGO ORIGINAL

Revista de Enfermagem do Centro-Oeste Mineiro

2021; 11/4159

DOI: http://doi.org/10.19175/recom.v10i0.4159

www.ufsj.edu.br/recom

\title{
Autocuidado de idosos com diabetes mellitus na perspectiva do modelo de atenção às condições crônicas
}

\author{
Self-care of older adults with diabetes mellitus from the perspective of the chronic care model \\ Autocuidado de ancianos con diabetes mellitus desde la perspectiva del modelo de atención de \\ condiciones crónicas
}

\section{RESUMO}

Objetivo: Conhecer as práticas de autocuidado de idosos com diabetes mellitus tipo 2. Método: Estudo qualitativo, que utilizou como base conceitual o Modelo de Atenção às Condições Crônicas, que contou com a participação de 12 idosos com diabetes. Utilizou-se, para a coleta de dados, entrevista individual e grupo focal. Foi utilizada a análise de conteúdo de Bardin. Resultados: Os idosos conhecem os desfechos da doença não controlada; a alimentação adequada é uma preocupação; muitos conheciam os cuidados com os pés; o enfermeiro foi responsável pela educação em saúde. Conclusão: Ainda existem lacunas no conhecimento dos idosos com diabetes que repercutem no autocuidado na perspectiva do Modelo de Atenção às Condições Crônicas. Entretanto o enfermeiro é um profissional de referência para o apoio às práticas educacionais à essa população.

Descritores: Diabetes mellitus; Autocuidado; Idoso; Atenção Primária à Saúde; Enfermagem.

\section{ABSTRACT}

Objective: To know the self-care practices of older adults with diabetes mellitus type 2 . Method: This is a qualitative study using the Chronic Care Model as a conceptual basis, in which 12 older adults with diabetes participated. Individual interviews and focus groups were conducted for data collection. Bardin's content analysis was used. Results: The older adults know the damage of not monitoring the disease; adequate diet is a concern; many know about foot care; the nurse was responsible for health education. Conclusion: There are still gaps in the knowledge of the older adults with diabetes that impairs self-care from the perspective of the Chronic Care Model. However, the nurse is a professional reference to support educational practices for this population.

Descriptors: Diabetes mellitus; Self Care; Aged; Primary Health Care; Nursing.

\section{RESUMEN}

Objetivo: Conocer las prácticas de autocuidado de las personas ancianas con diabetes mellitus tipo 2. Método: Estudio cualitativo, que utilizó como base conceptual el Modelo de Atención de Condiciones Crónicas, el cual contó con la participación de 12 ancianos diabéticos. Se utilizaron entrevistas individuales y de grupos focales para la recolección de datos. Se utilizó el análisis de contenido de Bardin. Resultados: Los ancianos conocen los resultados de la enfermedad no controlada; existe también preocupación por una nutrición adecuada; muchos sabían sobre el cuidado de los pies; la enfermera era responsable de la educación para la salud. Conclusión: Aún existen lagunas en el conocimiento de los ancianos con diabetes que afectan el autocuidado desde la perspectiva del Modelo de Atención de Condiciones Crónicas. Sin embargo, la enfermera es una profesional de referencia para el apoyo de las prácticas educativas de esta población.

Descriptores: Diabetes mellitus; Autocuidado; Anciano; Atención Primaria de Salud; Enfermería.
Francielle Renata Danielli Martins Marques ${ }^{1}$

0000-0002-8578-9615

Samile Bonfim de Oliveira ${ }^{1}$

0000-0002-4094-6410

Lígia Carreira $^{1}$

0000-0003-3891-4222

Cremilde Aparecida Trindade

Radovanovic $^{1}$

0000-0001-9825-3062

Sônia Silva Marcon ${ }^{1}$

0000-0002-6607-362X

Maria Aparecida Salci ${ }^{1}$

0000-0002-6386-1962

${ }^{1}$ Universidade Estadual de Maringá, PR, Brasil.

Autor correspondente:

Francielle Renata D. M. Marques E-mail: franrenata.martins@gmail.com

Como citar este artigo:

Marques FRDM, Oliveira SB, Carreira L, et al. Autocuidado de idosos com diabetes mellitus na perspectiva do modelo de atenção às condições crônicas. Revista de Enfermagem do CentroOeste Mineiro. 2021;11:e4159. [Access___ ]; Available in:__. DOI: http://doi.org/10.19175/recom.v10i0.4 159 
2| Marques FRDM, Oliveira SB, Carreira L, et al.

\section{INTRODUÇÃO}

O diabetes mellitus (DM) é uma condição crônica de alta morbidade, considerado uma epidemia que afeta mais de 463 milhões de pessoas no mundo e está associado a um risco aumentado no desenvolvimento de complicações agudas e crônicas ${ }^{(1)}$. O DM tipo 2 (DM2) é o responsável por $90 \%$ de todos os casos da doença, acometendo, aproximadamente, 425 milhões de pessoas no mundo(2).

O crescente aumento da expectativa de vida, quando combinado aos fatores de risco, faz com que o DM2 tenha maior incidência na população idosa estando relacionado a um maior risco de morte prematura por associação às fragilidades e síndromes geriátricas ${ }^{(2-3)}$.

A hiperglicemia persistente, ao longo do tempo, causa danos micro e macrovasculares, como a retinopatia, nefropatia, doença coronariana, doença cerebrovascular, doença arterial periférica e neuropatia periférica ${ }^{(1-2)}$. Diante das diversas possibilidades de complicações, torna-se imprescindível que os idosos com DM2 alterem seus hábitos de vida, com adesão ao tratamento medicamentoso, alimentação saudável e atividade física regular, requisitos mínimos para manter um bom controle metabólico ${ }^{(4)}$.

Para a orientação de mudanças de hábitos e apoio para a prática diária do autocuidado ao idoso com DM2, destaca-se o papel dos profissionais de saúde, ao promoverem atividades educativas, visando ao empoderamento das pessoas com essa condição crônica, para serem capazes de gerenciar seus próprios determinantes de saúde ${ }^{(3,5)}$.

A educação em saúde é uma importante estratégia ao estímulo ao autocuidado, pois desenvolve a conscientização sobre a doença e torna os envolvidos protagonistas dos cuidados, além de orientá-los, para que exerçam com autonomia as mudanças de hábitos de vida, contribuindo para a manutenção do seu bemestar $^{(6)}$. Ações educativas com foco no protagonismo da pessoa diante de sua condição crônica são coerentes e reforçam os princípios do Modelo de Atenção às Condições Crônicas (MACC), cujas estratégias ao autogerenciamento envolvem o autocuidado apoiado pelos profissionais de saúde ${ }^{(3,5,7)}$.

O autocuidado apoiado tem o propósito de utilizar uma nova abordagem à educação em saúde das pessoas com condições crônicas, com a utilização de tecnologias leves, pautadas nas relações de vínculo e acolhimento, as quais propõem a coparticipação dos sujeitos no estabelecimento de metas de cuidado $^{(7)}$. A Atenção Primária à Saúde (APS), que funciona como porta de entrada para a população e está articulada aos demais pontos de atenção à saúde, consolida a construção conjunta das práticas de autocuidado com iniciativas de promoção da saúde, para as pessoas com DM2, prevenção de agravos, recuperação e reabilitação de complicações existentes ${ }^{(7-8)}$.

A reorganização das práticas assistenciais, nos serviços de saúde, para o atendimento utilizando o autocuidado apoiado, conforme proposto pelo MACC, são implementadas gradativamente. Contudo alguns serviços de assistência às pessoas com DM2 ainda refletem um modelo curativista, sem comprometimento com condutas iatrogênicas, promovendo uma assistência baseada no estabelecimento da queixa, encaminhamentos desnecessários que, consequentemente, contribuem para o agravamento das complicações crônicas da doença ${ }^{(9)}$.

É essencial que o cuidado às pessoas acometidas pelo DM2 seja planejado, considerando determinantes e condicionantes em saúde, bem como as necessidades individuais e familiares, corroborando com os princípios da APS, com o modelo de saúde vigente, garantindo um atendimento resolutivo, acessível e longitudinal, respeitando a hierarquização e descentralização político-administrativa dos serviços $^{(8)}$.

Considerando o acompanhamento das pessoas com DM2 na APS, segundo o MACC, as ações dos profissionais de saúde devem estar sistematizadas e direcionadas, para a prevenção, a identificação e manejo das complicações crônicas, conduzidas por equipe capacitada para o enfrentamento desses eventos complexos ${ }^{(9)}$.

Apesar das práticas de autocuidado serem imprescindíveis, para impactar positivamente na qualidade de vida dos idosos com DM2 e no processo saúde-doença, ressalta-se a grande quantidade de estudos que descrevem a epidemiologia do DM2 e a pequena atenção dispensada às suas complicações e à necessidade de autocuidado ${ }^{(10)}$. Além disso, estudos ${ }^{(4,9,11-12)}$ apresentaram lacunas, nas práticas de autocuidado, para o enfretamento do DM2, apontando a necessidade de pesquisas que subsidiem os profissionais de saúde a 
aprimorarem suas ações educacionais e intervenções.

Nesse sentido, a relevância deste estudo consiste na importância de se investigar as práticas de autocuidado dos idosos com diagnóstico de DM2 que participam de atividades educativas individuais ou em grupo na APS. Os resultados contribuirão para o fortalecimento da promoção da saúde, com enfoque no autocuidado e prevenção de agravos em idosos com DM2, possibilitando a qualificação das estratégias educativas realizadas pelos profissionais de saúde. Além disso, contribuirá para encorajar a utilização de elementos que compõem o MACC na atenção às pessoas com condições crônicas na APS.

Diante dessas considerações, emergiu a seguinte pergunta de pesquisa: Como os idosos com DM2 reconhecem as práticas de autocuidado? Assim, este estudo teve como objetivo conhecer as práticas de autocuidado de idosos com diabetes mellitus tipo 2.

\section{MÉTODO}

Trata-se de um estudo descritivo, exploratório, de natureza qualitativa, que utilizou como base conceitual o Modelo de Atenção às Condições Crônicas $^{(7)}$. O cenário foi uma Unidade Básica de Saúde (UBS), localizada na região Sul do país, selecionada por se destacar no processo de tutoria regional para selo bronze.

A população do estudo foi composta por idosos com diagnóstico de DM2 assistidos na UBS. Foram incluídos no estudo pessoas que atendiam os seguintes critérios de elegibilidade: ter idade igual ou superior a 60 anos, possuir diagnóstico de DM2, estar estratificado como baixo, moderado ou alto risco, frequentar os atendimentos individuais e/ou em grupos oferecidos pela APS e participar do grupo focal. Os critérios de exclusão foram: apresentar condições físicas ou psicológicas que impossibilitassem participar da coleta de dados. Os participantes foram selecionados aleatoriamente à medida que compareciam na UBS.

A coleta de dados foi realizada no, período de outubro a novembro de 2019, mediante entrevistas individuais e grupo focal. A escolha pelo Grupo Focal se deu pela possibilidade de facilitar o diálogo, a interação e a troca de experiências entre os participantes, fatores fundamentais para compreender as práticas de autocuidado vivenciadas pelos participantes do grupo. Foram agendadas duas datas, para a realização das entrevis- tas individuais e, na sequência, o grupo focal, participando seis idosos em cada data, atendendo aos critérios de rigor dessa técnica metodológi$\mathrm{ca}^{(13)}$. A coleta de dados foi conduzida por dois pesquisadores enfermeiros, pós-graduandos e com experiência nas técnicas utilizadas, sendo um vinculado à UBS, o que facilitou a aproximação com os participantes e o estabelecimento de um diálogo produtivo durante os grupos focais.

Primeiramente, foi explicada a importância e os motivos de participarem da pesquisa e realizadas as entrevistas individuais, a partir de questionário semiestruturado que versava sobre aspectos sociais e clínicos (sexo; idade; tempo de diagnóstico; polifarmácia; avaliação dos pés e classificação do controle metabólico), com duração média de 15 minutos. Na sequência, os participantes se reuniram em semicírculo, para o início do grupo focal, no qual os pesquisadores assumiam as posições de moderador e observador ${ }^{(13)}$, sendo que o pesquisador vinculado à UBS assumiu a função de observador para diminuir os conflitos de interesse. A duração dos grupos focais foi de aproximadamente 70 minutos e o conteúdo foi audiogravado.

A questão norteadora dos grupos focais foi: Como você realiza o autocuidado para controle do diabetes e prevenção de complicações? E, na sequência, indagou-se sobre as mudanças de hábitos adotadas, realização de atividade física, alimentação saudável, cuidado com os pés. E qual profissional de saúde foi responsável pelas orientações e apoio ao autocuidado.

Todas as entrevistas e o conteúdo audiogravado obtido nos grupos focais foram transcritos na íntegra e organizados em um documento no Microsoft Word. Em seguida, foram submetidos à análise de conteúdo(14), seguindo as etapas de pré-análise, exploração do material, tratamento dos dados e inferência dos resultados. Na pré-análise, foi realizada leitura flutuante visando obter impressões iniciais sobre o material a ser analisado. Posteriormente, foram feitas leituras exaustivas do material com o intuito de explorar os dados obtidos. Na sequência, as mensagens foram codificadas e, em seguida, apreendeu-se o núcleo de sentido, agrupando-os por semelhanças em categorias temáticas.

A análise permitiu a elaboração de quatro categorias temáticas: a primeira foi composta por códigos relacionados ao conhecimento acerca das principais complicações do DM2, no qual os participantes revelaram que o conhecimento adquirido era um estímulo para a adesão ao 
4| Marques FRDM, Oliveira SB, Carreira L, et al.

autocuidado. Os códigos mais predominantes relacionados às práticas de autocuidado constituíram a segunda e a terceira categoriais. E, por fim, outros códigos revelaram o profissional que atuava como maior responsável por apoiar o autocuidado das pessoas com DM2, formando a última categoria do estudo.

A pesquisa foi aprovada pela Comissão Permanente de Avaliação de Projetos e do Comitê Permanente de Ética em Pesquisa com Seres Humanos (COPEP), conforme parecer $n^{\circ}$ 3.760.510 de acordo com as Resoluções 466/2012 e 510/2016 do Conselho Nacional de Saúde sobre pesquisas com seres humanos. Todos os participantes assinaram o Termo de Consentimento Livre e Esclarecido (TCLE) e, para preservar o sigilo e o anonimato, utilizou-se, para identificação, a letra "P", significando participante, seguido dos números de 1 a 12, para classificar a ordem das falas, por exemplo: P1, P2 ... a P12.

Utilizou-se o Consolidated Criteria for Reporting Qualitative Research (COREQ) para nortear o desenvolvimento do estudo.

\section{RESULTADOS E DISCUSSÃO}

Participaram do estudo 12 idosos com DM2, na faixa etária entre 60 e 79 anos, sendo oito mulheres, sete idosos com até cinco anos de diagnóstico da doença, seis em uso de polifarmácia, com consumo diário de cinco ou mais medicamentos; seis nunca foram submetidos ao exame dos pés por profissionais de saúde; seis cujo exame dos pés não apresentou riscos e sete com classificação de controle metabólico ruim. 0 controle metabólico ruim representa uma hemoglobina glicada $\geq 9 \%$ ou $\geq 7 \%$, na presença de complicações crônicas( $^{(2)}$, indicando maior necessidade de acompanhamento profissional das complicações existentes e prevenção do surgimento ou do agravamento de outras complicações.

Nos relatos, identificaram-se vários aspectos que interferiram no cuidado à saúde, os quais foram organizados nas seguintes categorias: Conhecimento sobre as complicações causadas pelo DM2; Práticas de autocuidado em relação aos hábitos de saúde; Práticas de autocuidado em relação aos pés e Profissional responsável pela educação em saúde.

\section{Conhecimento sobre as complicações causadas pelo DM2}

Essa categoria aborda o conhecimento prévio dos participantes a respeito das possíveis complicações do DM2. Ao questionar os participantes sobre as práticas de autocuidado, eles revelaram exercê-las para estabilização da doença e prevenção de complicações. Os resultados demonstraram que os idosos conhecem os desfechos da doença não controlada, o que indicou intervenções educativas bem sucedidas realizadas pela equipe de saúde local no âmbito do conhecimento das complicações.

"Meu irmão ficou cego por causa do diabetes, então eu tenho medo" (P3). "Quando prejudica os rins aí já não tem mais o que fazer" (P4). "Diabetes faz você cortar os pedaços do corpo" (P6). "Quem tem complicações é quem tem o diabetes mal controlado" (P7).

Para os participantes, o conhecimento das complicações do DM2 se constituiu em elemento influente para que as práticas de autocuidado fossem aderidas. As complicações micro e macrovasculares, como alterações oftalmológicas, de sensibilidade, renais e amputações também foram citadas em outro estudo com pessoas com $D M 2^{(5)}$. É imprescindível que os profissionais de saúde que atuam na APS atenham-se a todas as possíveis complicações crônicas do DM2, com adoção de intervenções proativas e multiprofissionais necessárias para a prevenção dessas complicações ${ }^{(9)}$.

As condutas profissionais, para a prevenção das complicações, precisam considerar os aspectos comportamentais individuais e o conhecimento do indivíduo sobre o curso natural da doença. As intervenções, quando associadas aos domínios comportamentais e psicossociais, favorecem a elaboração de planos de cuidados que contemplem as reais necessidades de saúde dessas pessoas, promovendo melhor qualidade de vida ${ }^{(15)}$.

A prevenção do DM2 e de suas complicações é hoje prioridade de saúde pública e necessita de novas estratégias para enfrentar esses obstáculos. Com o adequado monitoramento das pessoas na APS, os níveis de prevenção devem orientar essa prática. $\mathrm{Na}$ prevenção primária, a orientação objetiva eliminar os fatores de risco e prevenir o início da doença; a prevenção secundária monitora as pessoas com 
diagnóstico de DM2 para prevenir complicações agudas ou crônicas; e, a prevenção terciária, visa à reabilitação ou limitação das incapacidades produzidas pelas complicações instaladas ${ }^{(2,16)}$. Há ainda o conceito de prevenção quaternária, mencionado pela primeira vez por um médico da família, na Bélgica, que consiste em reduzir a medicalização e intervencionismo desnecessários, prevenindo danos iatrogênicos, apresentando-se como uma necessidade de aplicação na APS ${ }^{(17)}$.

$O$ adequado rastreamento das pessoas com condições crônicas na APS, conforme o MACC, visa priorizar os processos de saúde, para reduzir a incidência de intervenções desnecessárias (iatrogenia), a partir de condutas profissionais baseadas em protocolos e diretrizes clínicas ${ }^{(7)}$.

Incentivar a educação das pessoas com condições crônicas, para mudanças de comportamento com suporte para $\mathrm{O}$ autocuidado $^{(7)}$ é um dos principais investimentos em saúde pública, já que, quanto maior o conhecimento adquirido, maior é a melhora registrada na prática de autocuidado ${ }^{(16)}$. Trabalhar a prevenção das complicações crônicas do DM2 requer um envolvimento da própria pessoa e apoio da sua família, rede social, profissionais de saúde, gestores, serviços de saúde, sistema de saúde, em todos os níveis de atenção e políticas públicas favoráveis e aplicáveis, para que se possam atingir resultados satisfatórios para a vida daqueles que convivem com essa doença ${ }^{(9)}$.

Informar as pessoas com DM2 que elas possuem uma condição crônica, que não pode ser curada, mas, sim controlada, auxilia no entendimento e na adesão às mudanças de estilo de vida para evitar a progressão da doença e o surgimento de complicações ${ }^{(18)}$. Por isso, o acompanhamento das condições crônicas na APS deve se sustentar nas estratégias de prevenção terciária e quaternária, para reduzir as complicações e seus impactos na qualidade de vida e o uso indiscriminado de medicamentos ${ }^{(19)}$.

Importante destacar que um conhecimento satisfatório, em relação às complicações causadas pelo $\mathrm{DM} 2$, não é o mesmo que ter atitudes de enfrentamento para evitar o seu aparecimento. Estudo $^{(4)}$ realizado com usuários da APS com DM2 encontrou que, apesar de quase metade dos participantes apresentarem um conhecimento satisfatório sobre o diabetes, $98 \%$ possuíam atitudes negativas para o enfrentamento da doença.

Por esse motivo, condutas profissionais à luz do MACC compreendem ações de orientação, quanto ao reconhecimento das complicações da doença e de apoio, para a adesão às boas práticas de saúde, com elaboração de planos de cuidados com metas individualizadas e pactuadas com as pessoas atendidas. Por isso, seguir o tratamento proposto, participar da condução do tratamento com definições de metas e apoio à resolução de problemas aumentam a confiança e a habilidade das pessoas em manejar seus determinantes de saúde, indicando uma corresponsabilidade entre pessoa e equipe de saúde pelos resultados obtidos $^{(20)}$.

\section{Práticas de autocuidado em relação aos hábitos de saúde}

Essa categoria compreendeu os cuidados gerais para o controle do DM2. Emergiram aspectos relacionados aos fatores de risco modificáveis, como alimentação inadequada, tabagismo e sedentarismo. Todos esses aspectos foram destacados pelos participantes, quando instigados a comentarem sobre os cuidados relacionados aos hábitos de saúde.

"Não fumar, não comer muito doce, não tomar refrigerante" (P1). "Cortar açúcar, o sal e a carne gorda" (P8). "Não comer muito pão e bolacha doce" (P2). "Cortar o açúcar e a massa bran$c a$ " (P12). "Eu participei do grupo de tabagismo e consegui reduzir a quantidade de cigarros que fumo" (P4). "Tem que caminhar. Eu caminho com meu marido de segunda a sexta" (P5).

Em ambos os grupos, os primeiros comentários foram relacionados à dieta, evidenciando que a alimentação adequada é uma grande preocupação dos idosos com DM2. Porém ressalta-se que, muitas vezes, a preocupação pela mudança de hábitos tem início após o recebimento do diagnóstico da doença. O estilo de vida saudável, com alimentação adequada e prática de atividade física, deveria fazer parte da orientação terapêutica dos profissionais de saúde para todas as pessoas. Inclusive, em alguns casos, pode ser obtido controle glicêmico adequado apenas com as mudanças no estilo de vida, sem a necessidade de terapia medicamentosa ${ }^{(1-2)}$.

Recomenda-se que as pessoas com DM2 e seus familiares participem de programas de educação nutricional desde a descoberta da doença, com discussão sobre a importância do autocuidado, tornando o indivíduo independente e empoderado para tomar decisões relacionadas à alimentação e ao controle metabólico ${ }^{(21)}$. 
As intervenções de enfermagem mais trabalhadas com pessoas com DM2 e hipertensão arterial (HA) são relacionadas à educação, quanto ao controle da doença com mudança de hábitos de vida, como, por exemplo, aconselhamento nutricional, incentivo à adesão à atividade física, cessação tabágica e orientações para terapia medicamentosa, estimulando a participação ativa da pessoa na elaboração do seu plano de cuidados, durante a consulta de enfermagem, destacando a sua corresponsabilidade para o êxito dos resultados almejados ${ }^{(15)}$.

Quanto ao tabagismo, nota-se um reconhecimento dos participantes sobre os malefícios do tabaco para o agravamento da sua condição crônica, incluindo o relato de uma pessoa sobre a participação no grupo de tabagismo na APS. É consenso na literatura que o consumo de cigarros por pessoas com DM2 influencia negativamente o controle da doença. Estima-se que o tabagismo, importante fator de risco modificável para o desenvolvimento de DM2, esteja diretamente relacionado a $10 \%$ de todos os casos de $\mathrm{DM}^{(2)}$.

Neste sentido, as estratégias utilizadas pelos profissionais de saúde, ao mesmo tempo em que reiteram os benefícios da cessação tabágica, para evitar as complicações nas pessoas comDM2, também, precisam estar dotadas de uma postura acolhedora para dar amparo e motivação a essas pessoas nos momentos da abstinência ${ }^{(22)}$.

A prática de atividade física, também citada pelos participantes, envolve o tratamento não medicamentoso, assim como a alimentação. Ambas as práticas de cuidado são consenso na literatura como necessárias para controle metabólico ${ }^{(1-2)}$. Estudo realizado com pessoas com HA e DM2 atendidas, conforme preconiza o MACC, na região Sudoeste do Paraná, encontrou que quase $60 \%$ delas não praticavam atividade física regularmente ${ }^{(11)}$. Outro estudo também realizado com idosos, majoritariamente do sexo feminino, relatou que, em razão de as mulheres despenderem uma quantidade de horas dedicadas aos cuidados domésticos, o tempo destinado para atividades físicas ficava insuficiente ${ }^{(23)}$.

O envelhecimento, associado à diminuição da atividade física, promove o acúmulo de gordura visceral que favorece o aumento da resistência à insulina ${ }^{(2)}$. Assim, a atividade física regular tem efeito benéfico em vários aspectos: melhora da capacidade cardiorrespiratória, da composição corporal (diminuição de massa gorda e aumento de massa magra), da massa óssea e da sensibilidade à insulina, além de promover o bemestar psicossocial(2). Dessa forma, o incentivo à prática regular de atividade física entre os idosos visa diminuir a incidência de morbidades relacionadas à senilidade e síndromes geriátricas incapacitantes ${ }^{(23)}$ com um plano de cuidados elaborado cooperativamente entre equipe da APS, atenção especializada, usuário e família ${ }^{(7)}$.

O tratamento não medicamentoso para o DM2, em especial, a alteração dos hábitos alimentares, acarreta um sofrimento psíquico importante para a pessoa que, por diversas vezes, repercute na sua autoestima(16). Essa situação pôde ser apreendida nos seguintes relatos:

"Quem tem diabetes não pode comer nada"(P2). "Eu só posso olhar pra comida, não posso comer" (P9). "Me tira o prazer de comer" (P11). "Eles [os profissionais de saúde] falaram que eu não posso comer doce, mas eu como" (P12).

Quando a pessoa com DM2 não adere ao tratamento, ela passa a entender que as ações dos profissionais de saúde são verdadeiras barreiras por não coincidirem com suas vontades $^{(5)}$. Uma das estratégias do MACC é utilizar ferramentas, para evidenciar o protagonismo da pessoa com condição crônica, estimulando a participação ativa na aprendizagem e na gestão da sua condição de saúde ${ }^{(3,5,7)}$. Uma vez empoderada, essa pessoa tem potencial para contribuir com a melhora de seus resultados psicológicos e clínicos, já que a adesão às práticas de autocuidado aumenta a sua autonomia quanto à sua capacidade de cuidar da própria saúde ${ }^{(3,16)}$.

Segundo O MACC, é necessário o envolvimento das pessoas com condições crônicas, no seu processo de cuidado, sendo essa uma estratégia fundamental e inovadora na assistência, haja vista que a pessoa com condição crônica precisa aprender a lidar diariamente com essa condição, utilizando as práticas de autocuidado por toda a vida ${ }^{(20)}$.

Para que as pessoas comDM2 se envolvam no processo de cuidado, é necessário que os profissionais de saúde tenham habilidade de estabelecer diagnósticos que abranjam aspectos biológicos, culturais, sociais, econômicos e psicológicos. O diagnóstico de uma doença crônica, como o DM2, modifica a vida da maioria das pessoas e pode acarretar o surgimento de sentimentos de angústia e desespero, diante da percepção do pouco controle acerca da própria 
vida, o que diminui a habilidade para agir e $\operatorname{pensar}^{(2)}$.

Uma das formas de compreender melhor os momentos de transformação da pessoa com condição crônica é a utilização do Modelo Transteórico de Mudança de Comportamento, que define cinco estágios de mudança percorridos pela pessoa, ao alterar o próprio comportamento: Pré-contemplação: quando a pessoa não tem consciência de determinado problema ou não the dá a devida importância para que se inicie mudança de hábitos; Contemplação: a pessoa identifica o problema, porém ainda não se vê apto a enfrentar a mudança; Preparação: momento de determinação para o processo de mudança, com a definição de estratégias para modificação do comportamento; Ação: é o estágio em que se concretiza a mudança de comportamento e Manutenção: momento de maior esforço para prevenir lapsos ou recaídas que leva a pessoa a retornar ao comportamento antigo ${ }^{(2,7)}$.

Entender os estágios do comportamento da pessoa com condição crônica é fundamental ao processo de educação em saúde, uma vez que a mudança de comportamento e adesão às práticas de autocuidado dependem da sua motivação em alterar hábitos de vida. Quando a educação é aliada à mudança de comportamento, ela é capaz de criar condições para promover a transformação desejada. A atenção às pessoas com condições crônicas, segundo o MACC, preconiza a utilização do Modelo Transteórico, para avaliar o estágio, em que a pessoa se encontra e assim, desenvolver atividades educativas adequadas objetivando uma intervenção bem sucedida ${ }^{(7)}$.

Dessa maneira, os profissionais de saúde e a pessoa atendida tornam-se sujeitos do processo de transformação, que aprenderão com as experiências de ambos, tornando o processo natural e capaz de promover mudanças ${ }^{(2,7)}$.

\section{Práticas de autocuidado em relação aos pés}

O autocuidado com os pés é uma prática muito importante a ser considerada ao tratamento de pessoas com DM2, pois se constitui em uma rotina diária que contribui significativamente para evitar futuras complicações $^{(4)}$. Nesta categoria, foi possível observar uma grande divergência de informações, quanto às práticas adequadas de autocuidado, pois metade dos participantes relatou ter realizado a consulta de enfermagem com avaliação dos pés e a outra metade não recebeu essa assistência, o que impactou diretamente no modo como realizavam os cuidados diários.

"Enxugar bem o vão do dedo pra não dar frieira" (P3). "Andar de calçado fechado, que eu mudei desde que me orientaram. Usava chinelo" (P1). "Eu fui de chinelo no postinho e a enfermeira já me falou que não era pra usar esse tipo de calçado" (P6). "Não pode tirar cutícula" (P4). "Eu já vi gente que fez ferida porque estava com o pé rachado" (P3). "Não pode cortar o calo, nós que temos diabetes se cortar o calo o pé cai" (P12). "Eu passo creme no pé na hora de dormir" (P11). "Não pode passar creme no meio dos dedos e tem que secar bem sequinho" (P9). "Nunca ninguém me falou nada... eu sinto calor [nos pés], achei que era da menopausa" (P10). "Quando meu pé está muito rachado eu esfrego ele no piso" (P8).

A preocupação de alguns idosos com o risco de amputação refletiu no conhecimento prévio sobre o mal prognóstico das ulcerações. Pessoas com DM2 com ulcerações nos membros inferiores e doença arterial periférica podem ter uma sobrevida menor de cinco anos, ou seja, uma evolução tão ruim quanto uma doença

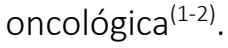

A busca por mais informações acerca das complicações relacionadas à falta de cuidado com os pés também foi constatada em outro estudo ${ }^{(24)}$, a partir de demanda dos próprios usuários, em encontros temáticos com os profissionais de saúde sobre cuidado com os pés das pessoas com DM2.

A neuropatia periférica leva à perda de sensibilidade, sendo um dos fatores de risco mais importantes, para o desenvolvimento de úlceras, principal causa para amputações em membro inferior nas pessoas com $\mathrm{DM}^{(1-2,12)}$. A hiperglicemia persistente pelo mau controle glicêmico é fator de risco para a neuropatia periférica(1-2).

Por isso, recomenda-se o exame dos pés, anualmente, para detectar sinais ou sintomas de perda de sensibilidade protetora e doença arterial periférica, com base na história e palpação de pulsos, mesmo em pessoas com DM2 sem úlceras ativas $^{(2)}$. Entre as recomendações de cuidado, as pessoas com DM2 não devem andar com os pés descalços, usar sapatos sem meias ou com chinelos de sola fina, seja em ambientes fechados ou ao ar livre, devem inspecionar diariamente os seus pés e o interior dos calçados, lavar os pés diariamente e secar bem entre os dedos, usar emolientes para lubrificar a pele seca e evitar 
agentes químicos ou qualquer outra técnica para remover calos ou hiperqueratose ${ }^{(2)}$.

Pessoas com DM2 que tiveram seus pés examinados periodicamente apresentaram maior sobrevida pelo fato de adotarem medidas de autocuidado, como hábitos de higiene e calçados adequados para prevenção de riscos$^{(12)}$. Esses achados mostram a necessidade de estender o acesso de atividades educativas a toda a população com DM2, para promover orientações adequadas e instituir o autocuidado apoiado, a fim de prevenir complicações ${ }^{(24)}$. Desse modo, o exame físico dos pés torna-se indispensável para toda a pessoa com diagnóstico de $\mathrm{DM} 2^{(1-2,12)}$.

Trabalhar com a educação em saúde, para o cuidado com os pés, é imprescindível, para a modificação de hábitos incompatíveis com as boas práticas de saúde. Os resultados apontaram que as pessoas que não tiveram seus pés examinados e não receberam orientações sobre esses cuidados tinham conhecimento sobre 0 autocuidado com os pés aquém dos demais que foram orientados, o que reforça a importância de promover a educação em saúde das pessoas com DM2 para o autocuidado com os pés.

Nesse sentido, ações educacionais podem ser trabalhadas, em grupos operativos, oficinas, atenção compartilhada em grupo, levando em consideração aspectos psicossociais e culturais que influenciam no contexto da aprendizagem ${ }^{(7,21)}$. Intervenções educativas, para o autocuidado de pessoas com DM2, elevaram o nível de conhecimento dessas pessoas sobre a doença, em relação àquelas que não participaram, representando a eficácia dessas ações ${ }^{(21)}$.

Segundo O MACC, as ações educativas precisam estar pautadas na escuta qualificada, na troca de experiências e no diálogo, construindo um ambiente no qual o usuário possa expressar dúvidas, sentimentos e queixas, com o objetivo de promover a autorreflexão e a autoavaliação das pessoas quanto aos comportamentos que se modificaram ao longo da prática educativa pela realização das metas propostas ${ }^{(24)}$.

Assim como os resultados encontrados em outro estudo ${ }^{(9)}$, ainda há dificuldade na atenção à saúde das pessoas com DM2 na APS, evidenciando lacunas quanto à implementação do proposto pelo MACC sobre o rastreamento das complicações crônicas.

\section{Profissional responsável pela educação em saúde}

A educação em saúde das pessoas com
DM2 na APS compreende uma relação mútua entre os profissionais de saúde e a pessoa com essa condição, em que ambos constroem conjuntamente o plano de cuidados, cujo papel do profissional é de instrumentalizá-la para que participe do seu tratamento de forma proativa. Compreende-se que essa relação entre profissional-usuário tem papel primordial nos reforços educativos sobre as práticas de autocuidado. A categoria destaca o profissional que mais realiza a educação em saúde dos idosos com DM2 e apoia o autocuidado para que a pessoa seja capaz de gerenciar seus determinantes de saúde.

"Ela [a enfermeira] fez os testes [nos pés] e depois me explicou tudinho... até como corta a unha" (P11). "Ela [a enfermeira] me explicou que minha glicemia glicada para estar boa tinha que ser abaixo de 7" (P3). "Ela [a enfermeira] me falou que eu preciso comer de três em três horas senão meu diabetes cai" (P7). "Eu aprendi a fazer o teste do dedo, ela [a enfermeira] me deu o aparelho, me ensinou a usar e a anotar os resultados pra levar no dia da consulta"(P8).

Destaca-se a unanimidade dos participantes em se referir o enfermeiro, como o profissional responsável pela educação em saúde que lhes permitiu conhecer as práticas de autocuidado. Esse resultado destaca o protagonismo do enfermeiro no momento, em que há uma mudança do modelo de assistência à saúde, que busca o rompimento da hegemonia do modelo biomédico. A vinculação da pessoa atendida ao profissional de saúde é fundamental ao processo de construção da aprendizagem, impactando positivamente nas medidas de autocuidado(6).

O enfermeiro inserido na APS é o profissional que mais tem contato e conhecimento das condições de saúde da sua população, por isso, relações de confiança com os usuários são comumente estabelecidas, ao longo do tempo, permitindo que as pessoas exponham sentimentos e problemas ${ }^{(25)}$. Quando o enfermeiro é adequadamente capacitado, torna-se mais fácil realizar os diagnósticos de enfermagem e, com o estabelecimento do vínculo adequado, implementar novas prescrições de cuidado potencializando a adesão ao tratamento proposto( ${ }^{(5)}$. Ressalta-se a importância de investimentos, na qualificação do enfermeiro, já que esse profissional é um dos principais responsáveis pela participação e apoio da pessoa na gestão da sua condição crônica ${ }^{(6)}$.

Consultas de enfermagem são consideradas 
como fatores de proteção para as pessoas com DM2 ao promover o autocuidado. Os enfermeiros têm a capacidade de realizar reforços educativos sobre cuidados com os pés, aplicação de insulina e hábitos saudáveis, bem como se mantêm disponíveis para o esclarecimento de dúvidas ${ }^{(12)}$. Acredita-se que a formação holística do enfermeiro, com o olhar direcionado para os aspectos biopsicossociais, intensifique a relação com a pessoa com DM2, já que essa é uma doença que causa um impacto negativo na vida dessas pessoas, necessitando de um cuidado mais amplo com amparo às reações emocionais e ao desenvolvimento do autocuidado ${ }^{(25)}$.

Entre todas as possibilidades de intervenções de enfermagem, a educação em saúde destaca-se como campo estratégico, para a promoção do autocuidado às pessoas com $\mathrm{DM} 2 \mathrm{e}$ evidenciam que o aconselhamento e o modelo educacional, aplicados na intervenção de enfermagem, são eficazes para melhorar 0 controle metabólico, bem como efeitos positivos na gestão do autocuidado ${ }^{(25)}$.

Contudo o processo terapêutico, para a atenção adequada às pessoas com DM2, precisa ser construído por uma equipe multiprofissional composta por assistentes sociais, educadores físicos, enfermeiros, farmacêuticos, médicos, nutricionistas, odontólogos, psicólogos, entre outros, sempre com a participação da pessoa com DM2, em todas as decisões, atuando de maneira ativa no seu tratamento(2).

A reorganização da APS, conforme o atual modelo de saúde, precisa que todos os profissionais de saúde incorporem os pressupostos do MACC e promovam uma assistência na perspectiva da autonomia e do empoderamento dos usuários $^{(20)}$. A unanimidade do profissional enfermeiro, em promover a educação em saúde, pode indicar uma prática dos demais membros da equipe multiprofissional meramente prescritiva, herança do modelo curativista.

As práticas educativas realizadas pelos profissionais atuantes, conforme o MACC, estão embasadas em três estilos de comunicação: escuta profissional qualificada; ação de direcionamento e propostas de alternativas para que a pessoa tome suas decisões ${ }^{(7)}$.

Como a formação dos profissionais de saúde ainda está fortemente centrada no modelo biomédico e tecnicista, é necessária a educação permanente para toda a equipe que atua, conforme o MACC, ampliando seus saberes, adotando uma comunicação efetiva e abordando novas estratégias para o atendimento às pessoas com condições crônicas ${ }^{(18)}$.

Ressalta-se que as atividades educativas, voltadas ao autocuidado, são desenvolvidas, em geral, pelos profissionais da equipe da APS, com intervenções contínuas objetivando fazer com que as pessoas conheçam mais profundamente suas condições crônicas de saúde para gerenciálas melhor ${ }^{(3)}$. Para isso, é importante que toda a equipe de saúde da APS implemente o autocuidado apoiado, com a utilização das ferramentas propostas pelo MACC.

\section{CONSIDERAÇÕES FINAIS}

Este estudo apresentou as práticas de autocuidado para o DM2 realizadas por idosos, permitindo identificar suas principais preocupações, quanto ao curso da doença, os cuidados diários, o reconhecimento dos problemas e o modo como buscam informações de saúde.

Os resultados demostraram as potencialidades que as ações educativas alcançaram, ao emergir aspectos relacionados ao conhecimento acerca das complicações relacionadas à doença e aos fatores de risco modificáveis, como alimentação inadequada, tabagismo e sedentarismo, demonstrando que investimentos nesse setor consolidam as boas práticas para o autocuidado apoiado. Ressalta-se que a identificação da dificuldade, em alterar os hábitos alimentares, repercutiu em sofrimento psíquico, sendo necessário que o profissional tenha um conhecimento prévio das tecnologias de saúde, proposta pelo MACC, para apoiar as pessoas com DM2 na prática do autocuidado. Os principais pontos de fragilidade identificados foram relacionados aos cuidados com os pés, indicando a necessidade da equipe de saúde fortalecer ações educacionais e avaliações, para o rastreamento de neuropatias periféricas, a fim de promover o autocuidado e prevenir complicações.

Considera-se que ainda existem lacunas no conhecimento dos idosos com DM2, que fragilizam as práticas de autocuidado, na perspectiva do MACC, que podem interferir no alcance da estabilização do DM2 e, consequentemente, contribuir para o aparecimento das complicações crônicas. Entende-se como potencialidade o estudo ter revelado o enfermeiro como um profissional de grande relevância, para o apoio ao autocuidado, na percepção dos idosos atendidos pela APS, 
10| Marques FRDM, Oliveira SB, Carreira L, et al.

fazendo alusões ao rompimento da hegemonia do modelo biomédico no que concerne às práticas de cuidado. Contudo há a necessidade de verificar o envolvimento dos demais profissionais da equipe com as estratégias de atenção, propostas pelo MACC, no que compete às ações de educação em saúde para as pessoas com DM2.

Como contribuições, para o avanço da enfermagem e da ciência, a identificação do modo como os idosos compreendem a doença e gerenciam suas escolhas diariamente pode auxiliar no planejamento e desenvolvimentos de intervenções profissionais, focalizadas nessa população e ancoradas na proposta do MACC, utilizando uma comunicação com escuta qualificada e sensível a reconhecer as fragilidades para o autocuidado, levando em conta as especificidades e experiências da pessoa idosa. Assim, o apoio ao autocuidado gerontológico pauta-se em aprimorar ações de educação em saúde como uma importante estratégia, para a conscientização sobre a doença e suas repercussões, tornando os envolvidos protagonistas do cuidado, com possibilidade de refletir positivamente para a saúde desta população.

Como limitações, a possibilidade de seus resultados estarem influenciados por características relativas ao contexto local das pessoas participantes, sendo importante a realização de estudos como este em outros cenários.

Ressalta-se o envolvimento positivo dos idosos participantes da pesquisa, o que permitiu ampla discussão das ações, na perspectiva da promoção do autocuidado e na postura acolhedora, que favoreceu expressarem os pontos de fragilidade, com destaque para o fato de muitos não terem sido submetidos à avaliação dos pés.

\section{REFERÊNCIAS}

1 - International Diabetes Federation. IDF Diabetes Atlas. 9th ed. [Internet]. Brussels, Belgium: International Diabetes Federation; 2019 [citado em 10 abr 2020]. Disponível em: https://www.diabetesatlas.org/en/

2 - Sociedade Brasileira de Diabetes. Diretrizes da Sociedade Brasileira de Diabetes 2019-2020. Organização Adriana Costa, FortiAntonio Carlos Pires, Bianca de Almeida Pittito. São Paulo. Editora: Clannad, 2019 [citado em 24 abr2020]. Disponível em: https://www.diabetes.org.br/profissionais/images LDIRETRIZES-COMPLETA-2019-2020.pdf

3 - Marques MB, Coutinho JFV, Martins MC, Lopes
MV de O, Maia JC, Silva MJ da. Intervenção educativa para a promoção do autocuidado de idosos com diabetes mellitus. Rev Esc Enferm USP 2019;53:e03517. DOI: 10.1590/s1980$\underline{220 \times 2018026703517}$

4 - Assunção SC, Fonseca AP, Silveira MF, Caldeira $A P$, Pinho $L$ de. Knowledge and attitude of patients with diabetes mellitus in Primary Health Care. Escola Anna Nery. 2017; 21(4): e20170208. DOI: 10.1590/2177-9465-ean-2017-0208

5 - Santos AL, Marcon SS, Teston EF, Back IR, Lino IGT, Batista VC, et al. Adherence to the treatment of diabetes mellitus and relationship with assistance in Primary Care. REME Rev Min Enferm. 2020;24:e-1279. DOI: 10.5935/1415$\underline{2762.20200008}$

6 - Teston EF, Sales CA, Marcon SS. Perspectivas de indivíduos com diabetes sobre autocuidado: contribuições para assistência. Escola Anna Nery. 2017; 21(2): e20170043. DOI: 10.5935/1414$\underline{8145.20170043}$

7 - Mendes EV. Os desafios do SUS. Brasília, DF: CONASS, 2019 [citado em 06 Dez 2020]. Disponível em:

https://www.conass.org.br/biblioteca/desafiosdo-sus/

8 - Teston EF, Spigolon DN, Maran E, Santos AL, Matsuda LM, Marcon SS. Nurses' perspective on health education in diabetes mellitus Care. Rev Bras Enferm. 2018;71(Suppl 6):2735-42. DOI: $\underline{10.1590 / 0034-7167-2018-0396}$

9 -Salci MA, Meirelles BHS, Silva DMVG. Prevention of chronic complications of diabetes mellitus according to complexity. Rev Bras Enferm. 2017;70(5):996-1003. DOI: 10.1590/0034-71672016-0080

10 - Gregg EW, Sattar N, Ali MK. The changing face of diabetes complications. Lancet Diabetes Endocrinol. 2016;4(6):537-47. DOI: 10.1016/S2213-8587(16)30010-9

11 - Piccoli C, Zonta FNS, Costa LD, Menetrier JV, Roque MS, Oliveira EM, et al. Epidemiological and clinical profile of accompanied patients in a model program for attention to chronic conditions. Cienc Cuid Saude. 2020; 19:e50327. DOI: $\underline{10.4025 / \text { cienccuidsaude.v19i0.50327 }}$

12 - Scain SF, Franzen E, Hirakata VN. Riscos 
associados à mortalidade em pacientes atendidos em um programa de prevenção do pé diabético. Rev Gaucha Enferm. 2018;39:e20170230. DOI: $\underline{10.1590 / 1983-1447.2018 .20170230}$

13 - Kinalski DDF, Paula CC, Padoin SMM, Neves $E T$, Kleinubing RE, Cortes LF. Focus group on qualitative research: experience report. Rev Bras Enferm. 2017;70(2):424-9. DOI: 10.1590/00347167-2016-0091

14 - Bardin L. Análise de Conteúdo. 3ạ reimpressão da 1a edição. São Paulo, Edições 70, 2016.

15 - Vieira VAS, Azevedo C, Sampaio FC, Oliveira PP, Moraes JT, Mata LRF. Cuidados de enfermagem para pessoas com diabetes mellitus e hipertensão arterial: mapeamento cruzado. Rev baiana enferm. 2017[citado em 19 Ago 2020];31(4):e21498. Disponível em: http://www.revenf.bvs.br/pdf/rbaen/v31n4/01025430-rbaen-31-4-e21498.pdf

16 - Almeida MS, Melo RHV, Vilar RLA, Silva AB, Melo ML, Júnior AM. Popular education in health with groups of diabetic elderly in the Family Health Strategy: an Action-Research. Rev Ciênc Plur. 2019; 5(2): 68-93. DOI: 10.21680/2446$\underline{7286.2019 v 5 n 2 I D 16954}$

17 - Jamoulle M. Quaternary prevention, an answer of family doctors to overmedicalization. Int J Health PolicyManag. 2015;4(2):61-64. DOI: 10.15171/ijhpm.2015.24

18 - Silocchi C, Junges JR, Martino A. Estudo comparativo das inovações sobre condições crônicas na atenção primária em Porto Alegre, RS, Brasil e Ferrara, Itália. Saúde soc. 2020;29(1):e190633. DOI: $10.1590 / \mathrm{s} 0104-12902020190633$

19 - Tesser CD, Norman AH. Geoffrey Rose e o princípio da precaução: para construir a prevenção quaternária na prevenção. Interface (Botucatu). 2019, 23,e180435, 2019. DOI: $\underline{10.1590 / \text { interface. } 180435}$

20 - Silva LB, Soares SM, Silva PAB, Santos JFG, Miranda LCV, Santos RM. Assessment of the quality of primary care for the elderly according to the Chronic Care Model. Rev Lat Am Enfermagem. 2018;26:e2987. DOI: 10.1590/1518$\underline{8345.2331 .2987}$
21 - Trajano SS, Martins LVM, Matos TNF, Falcão CSV, Bezerra LMM, Abdon APV, et al. Percepção de pacientes com diabetes sobre o autocuidado. Rev Bras Promoç Saúde, 31(3): 1-10, jul./set., 2018. DOI: $\underline{10.5020 / 18061230.2018 .7598}$

22 - Zampier VSB, Silva MH, Jesus RR, Oliveira PP, Jesus MCP, Merighi MAB. Manutenção da abstinência do tabaco por ex-fumantes: estudo fenomenológico. Rev Gaucha Enferm. 2017,38(4):e2017-0027. DOI: 10.1590/1983$\underline{1447.2017 .04 .2017-0027}$

23 - Silveira BDC, Kirchner RM, Dallepiane LB. Relação entre indicadores sociodemográficos e antropométricos e atividade física de homens e mulheres idosos. Cienc Cuid Saude. 2018;17(1). DOI: $10.4025 /$ cienccuidsaude.v17i1.36650

24 - Macedo MML, Cortez DN, Santos JC, Reis IA, Torres HC. Adherence to self-care practices and empowerment of people with diabetes mellitus: a randomized clinical trial. Rev Esc Enferm USP. 2017;51:e03278.DOI: $\quad$ 10.1590/S1980$\underline{220 \times 2016050303278}$

25 - Oliveira GYM, Almeida AMO, Girão ALA, Freitas CHA. Intervenções de enfermagem para promoção do autocuidado de pessoas com diabetes tipo 2: revisão integrativa. Rev Eletr Enf. 2016;18(1188). DOI: 10.5216/ree.v18.38691

\section{Editores Responsáveis:}

Patrícia Pinto Braga

Angélica Mônica Andrade

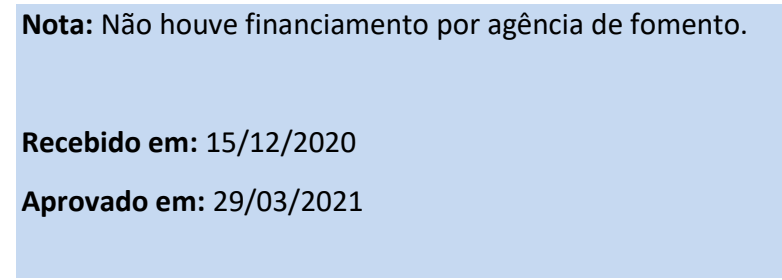

